\title{
Pitfalls in the management of indeterminate adrenal
}

\section{masses.}

Evangelia Vogiatzi, Panagiota Konstantakou, Xakousti Tzemeli, Antonis Sikiotis, Georgia Ntali

Department of Endocrinology and Diabetes, Alexandra General Hospital, Athens, Greece.

\section{Objectives}

A few adrenal masses may elude characterization on cross-sectional imaging and remain indeterminate. These include lipid-poor adenomas, adrenal metastases and carcinomas and phaeocromocytomas. It is important to distinguish between them, as phaeocromocytomas can be fatal, if operated without preoperative blockade.

\section{Methods}

Case report: A 63-years old woman presented with an incidentaloma of the right adrenal gland on an abdominal computed tomography scan performed because of chronic back and right subchondral pain.

She reported mild, non paroxysmic hypertension well controlled with a combined angiotensin-II receptor blocker/thiazide diuretic.

She had a cholecystectomy and bilateral hip arthroplasty 5 and 2 years ago.

\section{Results}

Her physical examination was unremarkable. Endocrine work-up with $1 \mathrm{mg}$ overnight dexamethasone test, aldosterone/renin ratio and urinary fractionated metanephrines and normetanephrines was normal.

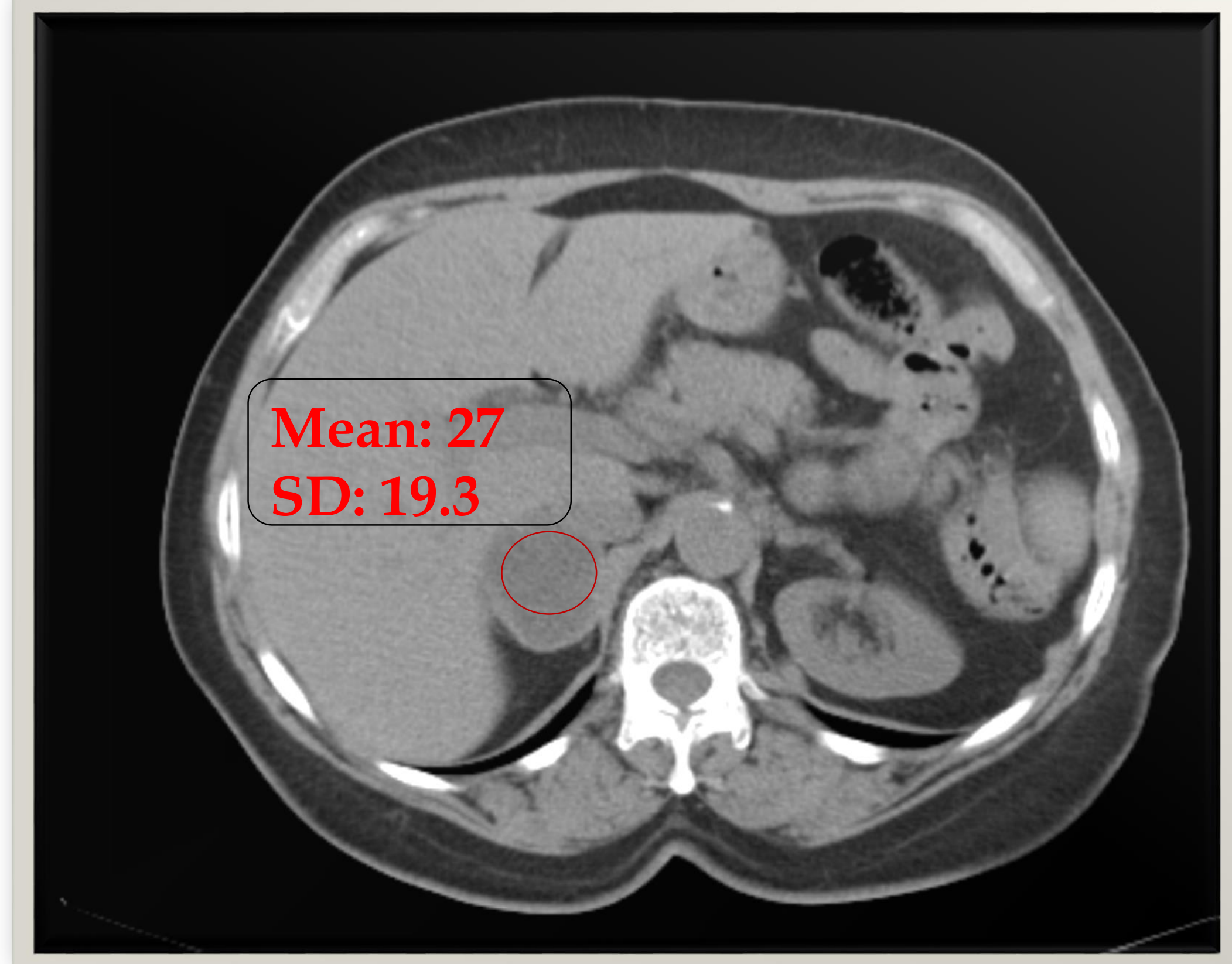

A. Non-contrast enhanced CT showed a round, well-defined right adrenal solid mass $4 \times 4 \times 4.4 \mathrm{~cm}$ with attenuation value $27 \mathrm{HU}$.

\section{Imaging}

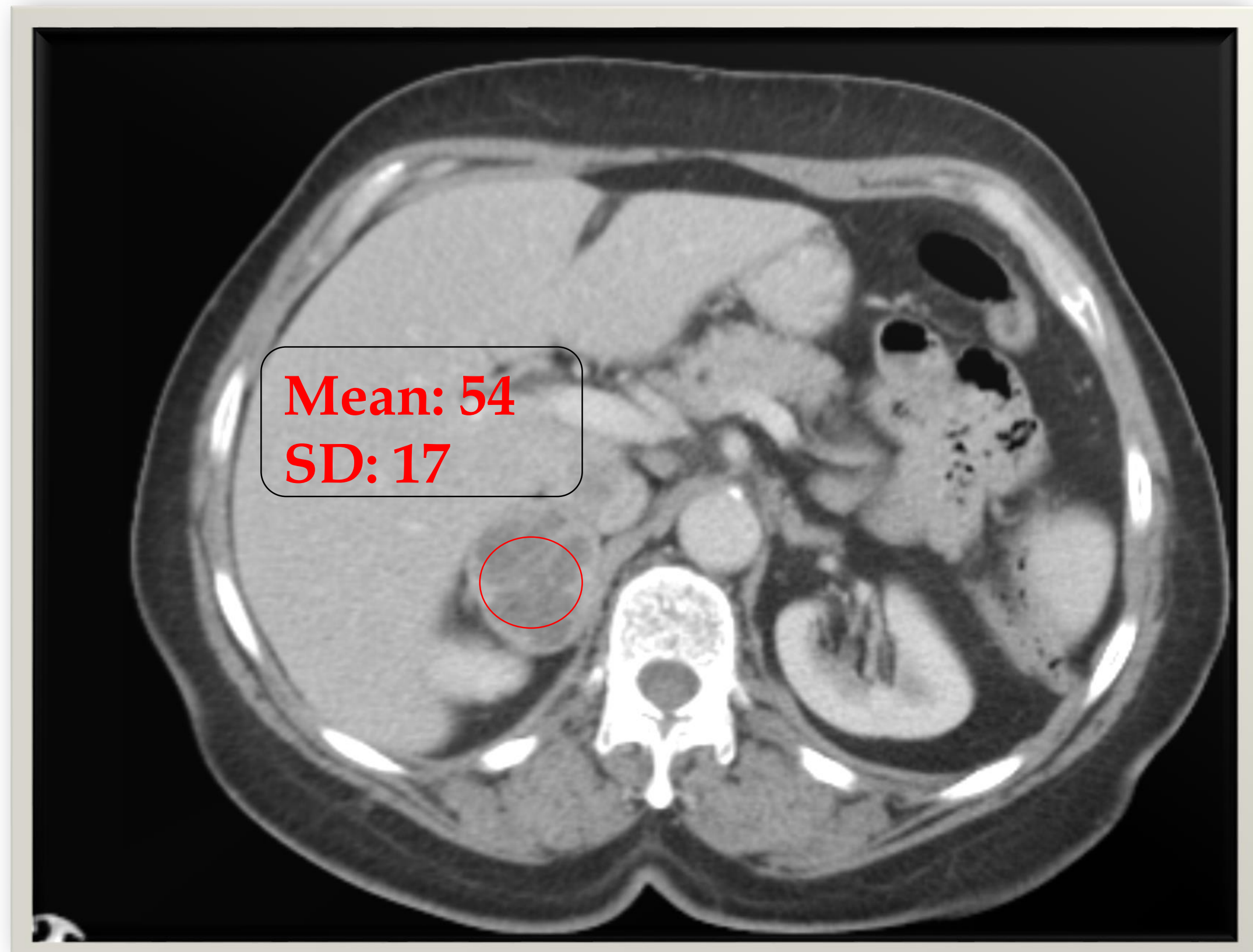

B and C. Contrast enhanced CT. Heterogeneous appearance with internal septa and central hypodense areas. Significant enhancement and no contrast washout in delayed phase.

Due to indeterminate nature surgery was decided. A laparoscopic approach was made and intraoperatively the patient developed acute hypertensive crisis successfully controlled with nitroprusside. Histopathology confirmed the diagnosis of a phaecromocytoma with Pass score 6.

\section{Conclusions}

This is a clinically and biochemically silent phaeocromocytoma which provoked an adrenergic spell during surgery. Malignant lesions and phaeocromocytomas may share common characteristics as intense enhancement and slower contrast washout. Suspicious radiologic findings should increase the medical alertness for the possibility of a catecholamine secreting tumour. Minimal handling of the tumour and perioperative vigilance to combat potential hypertensive crisis are the cornerstones in managing such cases.

\section{References}

- Fassnacht M, Arlt W, Bancos I et al. Management of adrenal incidentalomas: European Society of Endocrinology Clinical Practice Guideline in collaboration with the European Network for the study of Adrenal Tumors. Eur J Endocrinol. 2016 175:G1-G34.

- Mannelli M, Lenders JW, Pacak K, et al. Subclinical phaeocromocytoma. Best Pract Res Clin Endocrinol Metab. 2012 26:507-15.

- Sundahl N, Van Slycke S, Brusselaers N. A rare case of clinically and biochemically silent giant right pheocromocytoma: case report and review of literature. Acta Chir Belg. 2016116:239-242. 